\title{
Effect of erythropoietin on mesenchymal stem cell differentiation and secretion in vitro in an acute kidney injury microenvironment
}

\author{
N.M. Liu, J. Tian, W.W. Wang, G.F. Han, J. Cheng, J. Huang and \\ J.Y. Zhang \\ Department of Nephrology, The 455th Hospital of PLA, Shanghai, China \\ Corresponding author: J.Y. Zhang \\ E-mail: jinyuanzhangcn@126.com
}

Genet. Mol. Res. 12 (4): 6477-6487 (2013)

Received August 1, 2012

Accepted November 10, 2012

Published February 28, 2013

DOI http://dx.doi.org/10.4238/2013.February.28.14

\begin{abstract}
We investigated the effect of erythropoietin (EPO) on differentiation and secretion of bone marrow-derived mesenchymal stem cells in an acute kidney injury microenvironment. Acute kidney injury mouse models were prepared. Both renal cortices were then immediately collected to produce the ischemia/reperfusion kidney homogenate supernatant. The morphological and ultrastructural changes in the cells were observed using an inverted microscope and a transmission electron microscope. Cytokeratin-18 was detected using flow cytometry. Bone morphogenetic protein-7 levels, hepatocyte growth factor, and vascular endothelial growth factor in the culture medium were detected using an enzyme-linked immunosorbent assay. The cells had high CD29 and CD44 expression, as well as low CD34 and CD45 expression. More round and oval cells with cobble-like appearances were observed after EPO treatment. In addition, an increase in the number of rough endoplasmic reticula, lysosomes, and mitochondria was observed in the cytoplasm; the intercellular junction peculiar to epithelial cells was also seen on the cell surface. After treatment with ischemia/reperfusion kidney homogenate supernatant, cytokeratin-18 expression increased significantly and EPO could magnify its expression.
\end{abstract}


Bone morphogenetic protein-7 levels, hepatocyte growth factor, and vascular endothelial growth factor levels after treatment with ischemia/ reperfusion kidney homogenate supernatant significantly decreased, whereas EPO increased the cytokine secretion. The acute kidney injury microenvironment can induce the bone marrow-derived mesenchymal stem cells to partially differentiate into renal tubular epithelium-shaped cells, but weaken their secretion function. EPO intervention can boost up their differentiation function and reverse their low secretion effect.

Key words: Erythropoietin; Acute kidney injury; Cell differentiation; Cytokines; Bone marrow-derived mesenchymal stem cells

\section{INTRODUCTION}

Numerous studies have confirmed that bone marrow-derived mesenchymal stem cell (BM-MSC) transplantation helps to repair acute kidney injury (AKI). However, improvement is limited. Cell apoptosis during transplantation may be one of the reasons for the poor effectiveness of the treatment (Stolzing and Scutt, 2006; Hoffmann et al., 2010). Erythropoietin (EPO) is a glycoprotein that regulates the growth and differentiation of erythroid progenitor cells. EPO reportedly mediates the proliferation and apoptosis of a variety of non-hematopoietic cells through the erythropoietin receptor (EPOR). The surface of BM-MSCs also expresses EPOR (Zwezdaryk et al., 2007). Our previous study showed that EPO promotes the proliferation of mouse BM-MSCs (mBM-MSCs) and reduces their rate of apoptosis in vitro under AKI microenvironment conditions, effects that are mediated by EPOR (Liu et al., 2010). However, no pertinent studies can be found regarding the effect of EPO on AKI repair enhancement using BM-MSC transplantation. In the present study, the differentiation ability and secretory function of cultured mBM-MSCs and effects of EPO were studied to propose a method for enhancing AKI repair using BM-MSC transplantation.

\section{MATERIAL AND METHODS}

\section{Animals}

C57BL/6 mice were provided by the Experimental Animal Center of the Second Military Medical University (Animal Production License No. SCXK (Shanghai) 2007-0003). The animal models were established in the Experimental Animal Center of the Second Military Medical University [Animal Use Permit No. SYXK (Shanghai) 2007-0003].

\section{mBM-MSCs isolation}

Bone marrow was extracted from the femur and tibia of 4-week-old male C57BL/6 mice, and subjected to Percoll density gradient centrifugation and adherent culture to prepare the purified mBM-MSCs. The cells were digested with $0.25 \%$ trypsin and re-suspended for subculture. Cell growth was observed using an inverted microscope. Third generation mBM-MSCs were dissociated into a single-cell suspension, washed twice with phosphate-buffered saline (PBS), and adjusted to a sample concentration of $10^{6}$ cells $/ \mathrm{mL}$. CD29, CD44, CD34, and CD45 monoclonal 
antibodies (Abcam Inc.) and fluorescein isothiocyanate-labeled secondary antibodies (Jackson, Inc.) were all added to a $100-\mu \mathrm{L}$ sample, which was incubated in the dark at room temperature. The expression of the aforementioned antigens was detected using flow cytometry (FCM).

\section{Preparation of normal mouse kidney homogenate supernatant}

Fifteen male C57BL/6 mice were sacrificed, and both renal cortices of each mouse were immediately obtained. The renal cortices were cut into pieces on a clean bench after weighing, and then made into a $20 \mathrm{~g} / \mathrm{L}$ homogenate in a glass homogenizer in an ice bath using PBS as the homogenizing agent. The homogenate was then centrifuged at $20,000 \mathrm{rpm}$ for $15 \mathrm{~min}$ at $4^{\circ} \mathrm{C}$. The supernatant was filtered using a sterile disposable $30-\mu \mathrm{m}$ mesh filter and stored at $-70^{\circ} \mathrm{C}$.

\section{Preparation of ischemia/reperfusion (I/R) kidney homogenate supernatant}

The bilateral renal pedicles of 60 male C57BL/6 mice were clamped for $30 \mathrm{~min}$ and re-opened for $30 \mathrm{~min}$ to prepare the AKI models. Both renal cortices were immediately obtained and cut into pieces on a clean bench after weighing. The renal cortices were made into a $20 \mathrm{~g} / \mathrm{L}$ homogenate in a glass homogenizer in an ice bath using PBS as the homogenizing agent. The homogenate was then centrifuged at $20,000 \mathrm{rpm}$ for $15 \mathrm{~min}$ at $4^{\circ} \mathrm{C}$. The supernatant was filtered using a sterile disposable $30-\mu \mathrm{m}$ mesh filter and stored at $-70^{\circ} \mathrm{C}$.

\section{Experimental grouping}

Purified P3-mBM-MSCs were grown in different treatment groups on six-well culture plates at $10^{5}$ cells/well. The treatment groups were as follows: group A (control): low-glucose Dulbecco's modified Eagle's medium (DMEM) (glucose concentration: $1000 \mathrm{mg} / \mathrm{L}$ ) with $10 \%$ fetal bovine serum; group B (normal mouse kidney homogenate): low-glucose DMEM supplemented with $10 \%$ fetal bovine serum and normal mouse kidney homogenate supernatant, which was placed in a plug-in transformer on Petri dish (Figure S1); group C (I/R kidney homogenate): low-glucose DMEM supplemented with $10 \%$ fetal bovine serum and I/R kidney homogenate supernatant, which was placed in a plug-in transformer on Petri dish (Figure S1); group D (different EPO concentrations): low-glucose DMEM supplemented with $10 \%$ fetal bovine serum and I/R kidney homogenate supernatant with different EPO concentrations $(1,5$, 10, and $50 \mathrm{IU} / \mathrm{mL}$ for D1, D2, D3, and D4 subgroups, respectively) (Figure S1). Each group was incubated for $1,3,5$, and 7 days, respectively.

\section{Cell shape and ultrastructure}

The general changes in cell shapes in groups A, B, C, and D4 were observed under an inverted microscope on days 0,3 , and 7 . The cells of the aforementioned four groups, which were collected after digestion, were centrifuged, and the supernatants were discarded. The precipitate was rinsed three times with $0.1 \mathrm{M}$ phosphate buffer, fixed for an hour with $1 \%$ osmic acid, and rinsed for another three times with $0.1 \mathrm{M}$ phosphate buffer. In addition, the following procedures were conducted: block staining, dehydration in a graded acetone series, soaking, embedding, block trimming, positioning with a semi-thin section under a light mi- 
croscope, and sectioning with a microtome. The ultrastructure of the cells was examined in 5 fields chosen from each group using a transmission electron microscope (TEM). The results were obtained using the single binding method.

\section{Biological identification}

The mBM-MSCs from each group were digested using $0.25 \%$ trypsin, collected, and rinsed twice in PBS. Approximately $0.5 \mu \mathrm{L}$ CK18-FITC antibody was added to each tube. The mBM-MSCs were incubated for $30 \mathrm{~min}$ in the dark at $4^{\circ} \mathrm{C}$, and washed in PBS to remove the unreacted antibody. Finally, the cells were re-suspended in $300 \mu \mathrm{L}$ PBS and detected using FCM.

\section{Detection of BMP-7, HGF, and VEGF}

The cell supernatant from each group was collected at different times and stored at $-80^{\circ} \mathrm{C}$. Enzyme-linked immunosorbent assays on the collected supernatants were independently conducted according to the manufacturer protocols. The optical density (OD) values in each well were measured at 450,492, and $492 \mathrm{~nm}$ according to the instructions for the microplate reader. The standard OD was plotted against concentration to obtain the standard curve. The corresponding contents were obtained from the standard curve according to the OD value of samples.

\section{Statistical methods}

All measurement data were expressed as mean \pm standard deviation $(\bar{x} \pm \mathrm{s})$. One-way analysis of variance was used to determine significant differences between groups. The $t$-test was used to compare any two groups using the SPSS 13.0 statistical software.

\section{RESULTS}

\section{Isolation and identification of mBM-MSCs}

The C57BL/6 mouse BM-MSCs obtained after Percoll density gradient centrifugation and adherent culture were spindle-like, with lucent cytoplasm and clear nuclei. Based on the FCM results, cell surface CD29 expression was $99.71 \pm 0.13 \%$ and the positive rates for CD34, CD44, and CD45 were $1.63 \pm 0.09 \%, 99.14 \pm 0.69 \%$, and $0.74 \pm 0.15 \%$, respectively. The results were consistent with mBM-MSCs surface antigen characteristics, indicating that the P3-cells were relatively pure (Figure 1).

\section{BUN and Scr levels of mice with AKI}

The blood urea nitrogen (BUN) level of AKI mice was $13.80 \pm 4.14 \mathrm{mM}$, and the Scr level was $45.32 \pm 7.15 \mu \mathrm{M}$. Both were remarkably higher than those of normal mice (BUN, $5.71 \pm 0.62 \mathrm{mM}$; Scr, $25.80 \pm 6.94 \mu \mathrm{M} ; \mathrm{P}<0.01)$. The conformity with the diagnostic criteria of AKI indicated that the model was successfully established in the present study. The I/R kidney homogenate obtained from the cortex of both kidneys could be used to simulate the AKI microenvironment in vitro. 

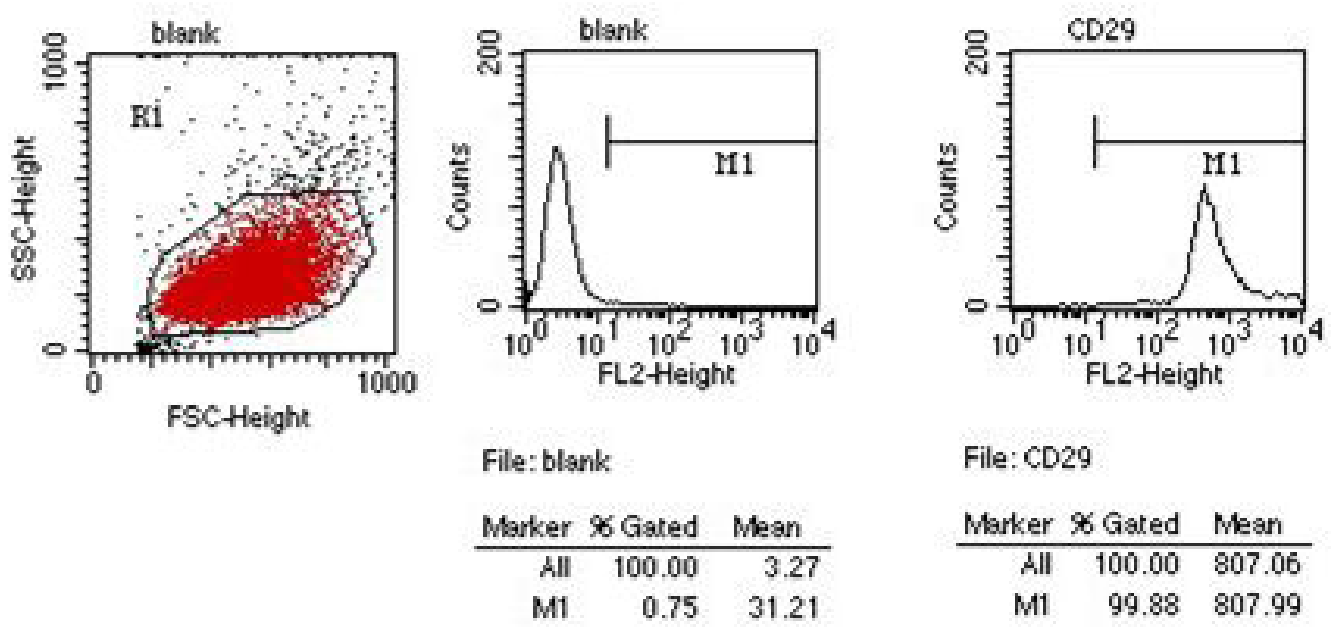

File: CD29
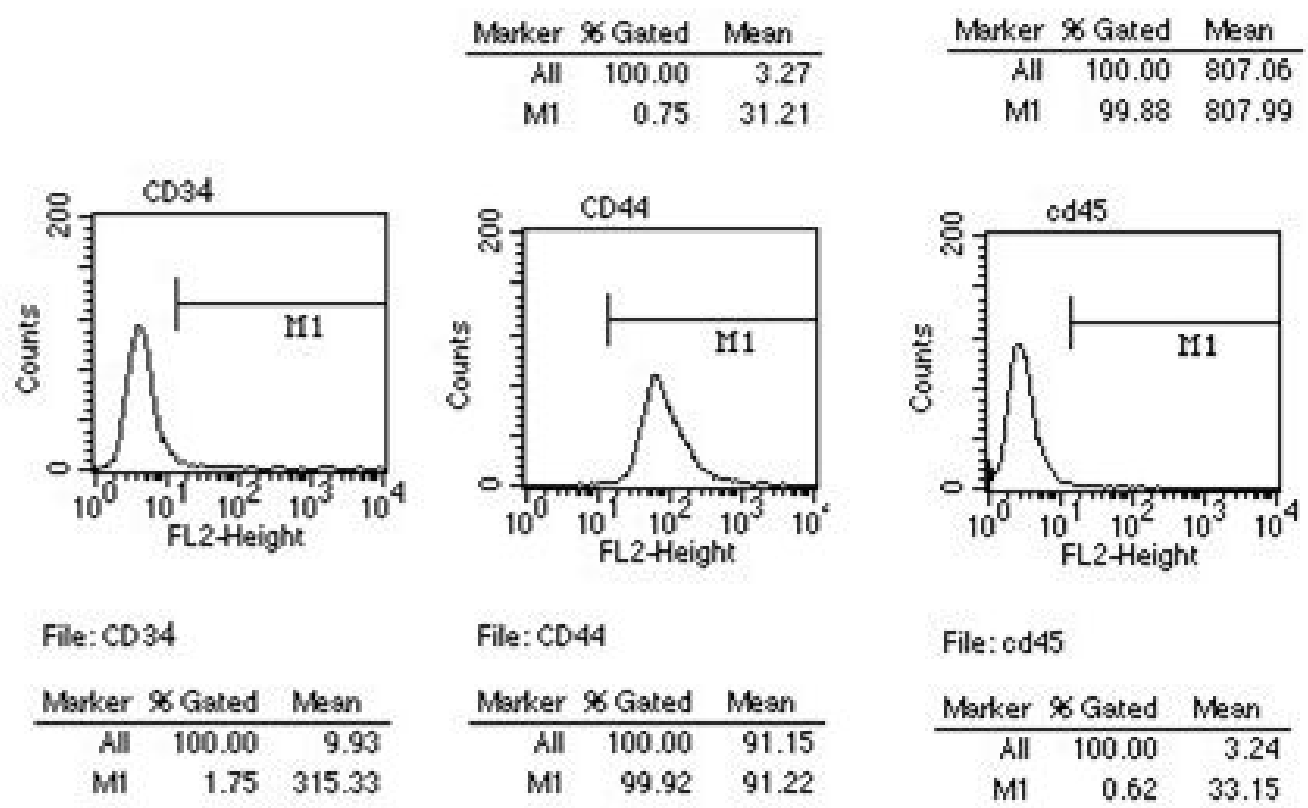

File: $C D 44$

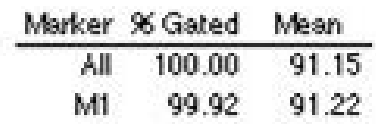

File: $c d 45$

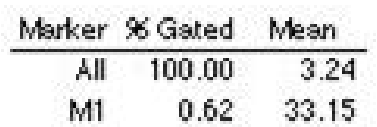

Figure 1. mBM-MSCs surface marker analysis via flow cytometry. The majority of the cells tested positive for CD29 and CD44, whereas a small portion tested positive for CD34 and CD45.

\section{General changes in cell shape}

The mBM-MSCs in groups A and B, which were observed under an inverted microscope, were found to grow along the plate wall in long spindles. Several mBM-MSCs in the culture plate in group $\mathrm{C}$ on day 3 were ellipsoidal and characterized by having short spindles. Many mBM-MSCs on day 7 were round and ellipsoidal and characterized by having short fat spindles. Moreover, the shape of many mBM-MSCs in group D4, which were treated with $50 \mathrm{IU} / \mathrm{mL}$ EPO, was round and ellipsoidal, and the cells had a cobblelike appearance (Figure S2). 


\section{Changes in ultrastructure}

The nuclei of mBM-MSCs grown in groups A and B, which were observed under an electron microscope, had increased in size. However, the other organelles had a relatively simple structure (Figure 2A). The ultrastructure of group B was the same as that of group A. In the cytoplasm of several mBM-MSCs induced with the I/R kidney homogenate, the amount of the granular endoplasmic reticulum, lysosomes and mitochondria significantly increased. The microvilli and intercellular junction peculiar to epithelial cells were also seen on the cell surface (Figure $2 \mathrm{~B}, \mathrm{C}$ and D). These apparent ultrastructural changes were also observed in the mBM-MSCs in group D4 with $50 \mathrm{IU} / \mathrm{mL}$ EPO.

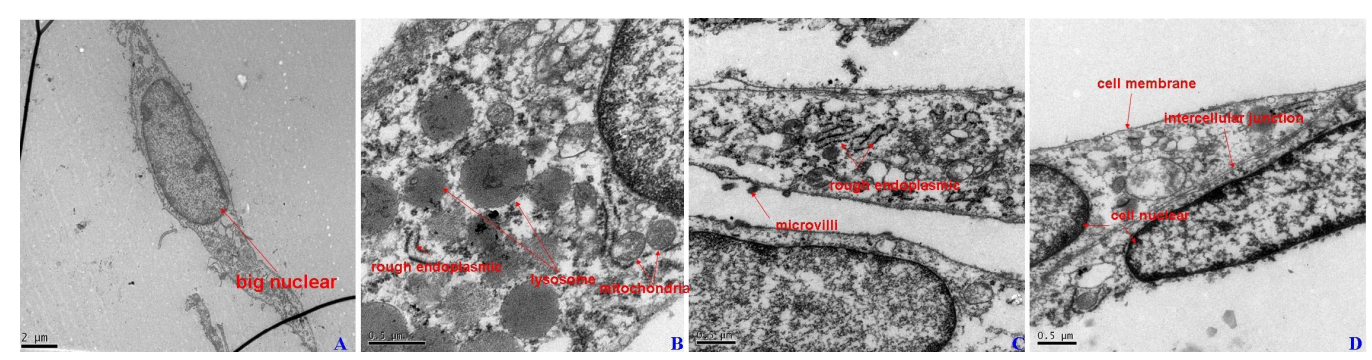

Figure 2. mBM-MSC ultrastructure of each group. A. Ultrastructure of group A (the nuclei of the mBMMSCs were bigger, few organelles were seen in cytoplasm), the ultrastructure of group B was the same to group A. B. C. D. Ultrastructure of group C (the granular endoplasmic reticulum, lysosome, and mitochondria significantly increased, and the microvilli and intercellular junction peculiar to epithelial cells were also seen on the cell surface), the ultrastructure of group D was the same to group C.

\section{Positive expression rate of CK18}

The CK18 content in the mBM-MSCs of Group A, which was determined using FCM, was found to be very small. No significant change in the positive expression rate of CK18 was observed after the treatments with normal mouse kidney homogenate supernatant. The positive expression rate of CK18 significantly increased after the treatment with the I/R kidney homogenate supernatant $(\mathrm{P}<0.01)$ compared with that of group B. CK18 expression was also shown to be time-dependent. Moreover, EPO treatment amplified the induction effect of the $\mathrm{I} / \mathrm{R}$ kidney homogenate on $\mathrm{mBM}-\mathrm{MSCs}$, which increased the expression rate in a concentration- and time-dependent manner $(\mathrm{P}<0.05$ or $\mathrm{P}<0.01)$ (Figure 3 ).

\section{Cytokine levels}

The BMP-7 content in the supernatant of group B at each time point slightly increased compared with that of group A, but the difference was not statistically significant. The BMP-7 content after treatment with the I/R kidney homogenate supernatant was statistically significantly decreased $(\mathrm{P}<0.05$ or $\mathrm{P}<0.01)$. The BMP-7 content increased after EPO treatment and, at some time points, was even close to that in groups A and B. The differences were statistically significant compared with group $\mathrm{C}$, which indicates that increase in BMP-7 content was concentration- and time-dependent (Figure 4A). 


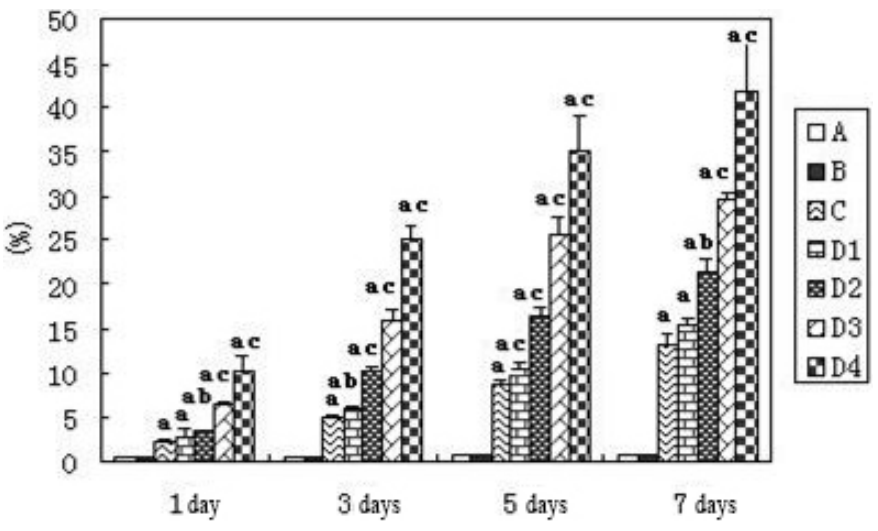

Figure 3. Positive expression rate of CK18 via flow cytometry. $\mathrm{A}=$ group $\mathrm{A}$ (control group); $\mathrm{B}=$ group $\mathrm{B}$ (normal mice kidney homogenate intervention group); $\mathrm{C}=$ group $\mathrm{C}$ [ischemia/reperfusion (I/R) kidney homogenate intervention group]; D1, D2, D3, and D4 = I/R kidney homogenate supernatant plus erythropoietin (EPO) and EPO concentrations were $1,5,10$, and $50 \mathrm{IU} / \mathrm{mL}$, respectively. Versus group $\mathrm{B}$ : ${ }^{\mathrm{P}}<0.01$; versus group $\mathrm{C}$ : ${ }^{\mathrm{b}} \mathrm{P}<0.05,{ }^{\mathrm{c}} \mathrm{P}<0.01$.

A

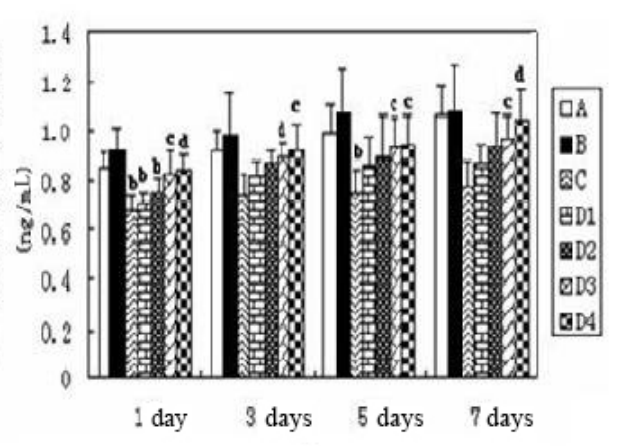

B

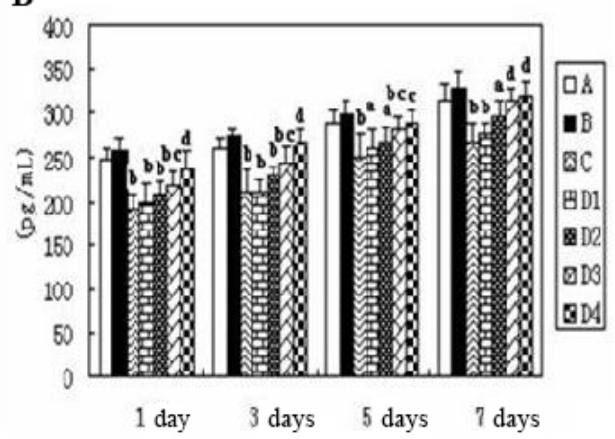

C

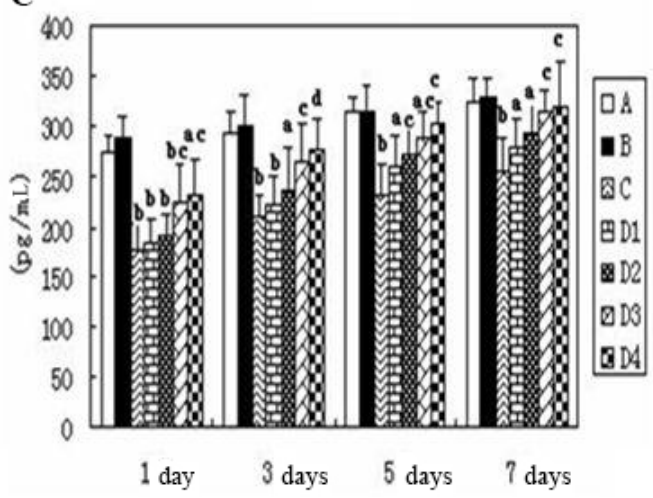

Figure 4. Cytokine expression in each group through ELISA. A. BMP-7 expression; B. HGF expression; C. VEGF expression. A = group A (control group); B = group B (normal mice kidney homogenate intervention group); $\mathrm{C}=$ group $\mathrm{C}$ [ischemia/reperfusion $(\mathrm{I} / \mathrm{R})$ kidney homogenate intervention group]; D1, D2, D3, and D4 = I/R kidney homogenate supernatant plus erythropoietin (EPO) and EPO concentrations were 1, 5, 10, and $50 \mathrm{IU} / \mathrm{mL}$, respectively. Versus group $\mathrm{B}$ : ${ }^{\mathrm{a}} \mathrm{P}<0.05,{ }^{\mathrm{b}} \mathrm{P}<0.01$; versus group $\mathrm{C}$ : ${ }^{\mathrm{P}} \mathrm{P}<0.05,{ }^{\mathrm{d}} \mathrm{P}<0.01$ 
The HGF content in the supernatant in group B at each time point slightly increased compared with that of group A, but the difference was not statistically significant. The HGF content significantly decreased at each time point after treatment with the I/R kidney homogenate supernatant $(\mathrm{P}<0.05$ or $\mathrm{P}<0.01)$. The HGF content at some time points remained much lower than that of group B after combined treatment with EPO. However, the content was significantly higher at the same time points compared with group $\mathrm{C}(\mathrm{P}<0.05$ or $\mathrm{P}<0.01$ at some time points), which indicates that the increase in HGF content was time-dependent (Figure 4B).

The VEGF content in the supernatant from group B at each time point slightly increased compared with that of group A, but the difference was not statistically significant. The VEGF content significantly decreased at each time point after treatment with I/R kidney homogenate supernatant $(\mathrm{P}<0.01)$. EPO treatment increased the VEGF content compared with group $\mathrm{C}$ and was even close to that in group $\mathrm{Ba}$ some time points $(\mathrm{P}<0.05$ or $\mathrm{P}<0.01)$ (Figure 4C).

\section{DISCUSSION}

AKI and its complications continue to be one of the main causes of mortality despite advancements in medicine. Apoptosis and necrosis of the renal tubular epithelial cells (RTECs) are the causes of AKI, and are involved in kidney injury protraction and development (Ortiz et al., 2002). Therefore, preserving the RTECs is crucial in AKI treatment.

Stem cell transplantation for AKI is an emerging technology in cell biological engineering, in which stem cells are transplanted into the damaged kidney and differentiate into RTECs or improve the partial microenvironment via their paracrine function, both of which consequently improve kidney function (Muro et al., 2008; Parra-Medina et al., 2009). BMMSCs are relatively ideal "seed cells" for transplantation. Numerous studies have demonstrated the differentiation and secretory functions of BM-MSCs (Anjos-Afonso et al., 2004; Semedo et al., 2007; Liu et al., 2009). The therapeutic safety and effectiveness of EPO for AKI patients have also been widely proven. However, no pertinent studies exist on the beneficial effects of EPO on BM-MSC transplantation into the kidneys, which leads to the enhancement of the transplant treatment effect. The present study initially investigated the differentiation and secretory functions of BM-MSCs to determine the effects of EPO on BM-MSCs in an AKI microenvironment.

In the present study, the morphological characteristics of mBM-MSCs cultured in vitro are in agreement with those reported in the literature. High CD29 and CD44 expressions and low CD34 and CD45 expressions were determined using cell phenotype analysis, and these results coincide with the mBM-MSC phenotype characteristics. Cultured RTECs are normally round or oval and grow on the walls of the container in a cobblestone-like arrangement. With regard to ultrastructure, several organelles, such as granular endoplasmic reticulum, lysosomes, and mitochondria can be observed in the cytoplasm. Treatment with an I/R kidney homogenate supernatant in a plug-in transformer on culture plate was performed to simulate the AKI microenvironment in vitro. The results revealed the morphologic characteristics of RTECs in the cultured mBM-MSCs via induction. CK18 expression of cultured mBM-MSCs was positive using FCM. CK18, a cytokeratin, can be used as an epithelial cell marker. The RTECs prioritize CK18 expression (Mao et al., 2004); thus, this specificity can be adopted to identify RTECs. The pore diameter of the semi-permeable membrane for the plug-in culture 
plate was $8 \mu \mathrm{m}$, which allows cytokines to freely permeate through the membrane but prevents cells from penetrating. Hence, the possibility of damaged RTECs and mBM-MSCs combining was eliminated. Moreover, mBM-MSCs differentiation into RTECs by induction was proven. In the present study, low CK18 expression was also observed in the mBM-MSCs in group A. This result was probably due to the heteropolarity in the 6-well plastic culture plates and the fact that fetal bovine serum in the culture medium contains substances, which may induce a slight change in phenotype in cells under a polar environment (Shahdadfar et al., 2005). The normal kidney homogenate supernatant was also used in a treatment group. The cells in this group did not undergo any morphological change and also had low CK18 expression. The reason is that differentiation of BM-MSCs needs stimulation from the cytokines secreted by damaged visceral organs. However, these cytokines are not secreted by the normal kidney tissues (Singaravelu and Padanilam, 2009).

In fact, the AKI microenvironment is adverse to the replication and multiplication of BM-MSCs in the kidney (Tang et al., 2005; Zhang et al., 2010). In previous studies, treatment with I/R kidney homogenate in vitro significantly decreased the capacity of mBM-MSCs to multiply. Thus, apoptosis and irreversible necrosis occur in many cells (Liu et al., 2010), which decrease the differential quantity of mBM-MSCs in an AKI microenvironment. EPO treatment induced the cultured cells to take on the morphological characteristics of RTECs, which causes an increase in CK18 expression. Increase in CK18 expression showed an obvious dose and time dependence when the EPO concentration was between 1 and $50 \mathrm{IU} / \mathrm{mL}$, which demonstrates that EPO enhances the effect of AKI microenvironment-induced mBMMSC differentiation. For the mechanism, it is probable that EPO combines with EPOR on the mBM-MSC surface and induces EPOR to form activated dimers and simultaneously activates the associated JAK2. Thus, EPO activates downstream signaling molecules, such as STAT-5, after phosphorylation of the EPOR tyrosine residue. This transduces the stimulation signals into the nucleus and regulates many types of EPO-mediated gene expression to promote mBM-MSC multiplication under AKI microenvironment conditions. Moreover, mBM-MSC apoptosis is prevented by regulating the caspase/ $\mathrm{Bcl}$ family components, which has been confirmed in previous experiments (Liu et al., 2010). Therefore, the quantity of mBM-MSCs that differentiate into RTECs significantly increases.

The kidney is one of the organs that are hypersensitive to I/R injury. The role of cytokines in organ $\mathrm{I} / \mathrm{R}$ injury has gained increased attention as a result of many studies on the cytokine network. BM-MSCs possess powerful secretory functions, which assist in AKI repair through their paracrine effect (Di Nicola et al., 2002; Singaravelu and Padanilam, 2009). In the present study, the supernatant of mBM-MSCs grown in low-sugar DMEM was found to contain the cytokines BMP-7, HGF, and VEGF, all of which can facilitate the repair of renal injury (Hruska, 2002). Supplementing BMP-7 during renal injury can alleviate renal interstitial fibrosis and protect the integrity and function of the renal structures (Zeisberg and Kalluri, 2008). HGF can increase the rate of mitosis in RTECs and promotes anti-apoptosis and hyperplastic functions (Dai et al., 2004). VEGF can reduce the quantity of apoptotic cells in the glomerulus and renal tubule in a renal infarction model (Suga et al., 2001). In the group treated with normal kidney homogenate supernatant, cytokine expression slightly increased because the kidney can also secrete microprotective cytokines which can be released into the supernatant through the semipermeable membrane of the plug-in culture plate. However, the difference between the two groups was not statistically significant. After treatment with 
the AKI homogenate, cytokine secretion remarkably decreased compared with group B, because the AKI homogenate inhibits the multiplication of $\mathrm{mBM}-\mathrm{MSC}$ and promotes apoptosis, which reduces the capacity of mBM-MSCs to secrete and regulate cytokines (Liu et al., 2010). Although BM-MSC can be used to treat damaged renal tissue through its paracrine function, the effect can be significantly weakened when BM-MSCs are transplanted to treat AKI. EPO treatment facilitates the multiplication of $\mathrm{mBM}-\mathrm{MSCs}$ in an AKI microenvironment and prevents apoptosis. Thus, EPO can enhance the secretory capacity of mBM-MSCs. The results demonstrated that after treatment with different EPO concentrations, the secretion of BMP-7, HGF, and VEGF increased by varying degrees compared with group $\mathrm{C}$, indicating that this effect is concentration- and time-dependent.

The results of the in vitro experiments demonstrated that EPO treatment enhances the differentiation of mBM-MSCs into RTECs under AKI microenvironment conditions. Moreover, EPO treatment also increased the secretory capability of mBM-MSCs. Therefore, EPO is suitable in AKI treatment using in vivo BM-MSC transplantation in terms of promoting differentiation and enhancing cytokine secretion. However, this conclusion needs to be further confirmed through in vivo animal experiments and clinical studies.

\section{ACKNOWLEDGMENTS}

Research supported by the PLA Nanjing Military Region 122 Program Leading Scholars Training Funds, the PLA Nanjing Military Medical Region Health Funds (\#07M023), the PLA Nanjing Military Region Technology Innovation Key Project Funds (\#09Z006), the Shanghai Municipal Health Bureau Research Projects Youth Fund (\#2009Y119), and the Shanghai Municipal Commission of Science and Technology Project Funds (\#11ZR1449600).

\section{Supplementary material}

\section{REFERENCES}

Anjos-Afonso F, Siapati EK and Bonnet D (2004). In vivo contribution of murine mesenchymal stem cells into multiple cell-types under minimal damage conditions. J. Cell Sci. 117: 5655-5664.

Dai C, Yang J, Bastacky S, Xia J, et al. (2004). Intravenous administration of hepatocyte growth factor gene ameliorates diabetic nephropathy in mice. J. Am. Soc. Nephrol. 15: 2637-2647.

Di Nicola M, Carlo-Stella C, Magni M, Milanesi M, et al. (2002). Human bone marrow stromal cells suppress T-lymphocyte proliferation induced by cellular or nonspecific mitogenic stimuli. Blood 99: 3838-3843.

Hoffmann J, Glassford AJ, Doyle TC, Robbins RC, et al. (2010). Angiogenic effects despite limited cell survival of bone marrow-derived mesenchymal stem cells under ischemia. Thorac. Cardiovasc. Surg. 58: 136-142.

Hruska KA (2002). Treatment of chronic tubulointerstitial disease: a new concept. Kidney Int. 61: 1911-1922.

Liu NM, Tiam J, Cheng J, Hu DY, et al. (2009). Mesenchymal stem cells homing to the injured kidneys and their function assay in mice. Chin. J. Nephrol. Dial. Transplant 18: 430-437.

Liu NM, Tiam J, Cheng J, Hu DY, et al. (2010). Differentiation, division and proliferation of cultured mesenchymal stem cells under acute kidney injury microenvironment. Chin. J. Nephrol. Dial. Transplant 19: 435-439.

Mao HJ, Wang XY, Xu CF and Cheng BG (2004). Primary culture, subculture and identification of human renal proximal tubule cells. Acta Univ. Med. Nanjing 24: 561-564.

Muro F, Iimura S, Yoneda Y, Chiba J, et al. (2008). Identification of 4-[1-[3-chloro-4-[N'-(5-fluoro-2-methylphenyl) ureido]phenylacetyl]-(4S)-fluoro-(2 S)-pyrrolidinylmethoxy] benzoic acid as a potent, orally active VLA-4 antagonist. Bioorg. Med. Chem. 16: 9991-10000.

Ortiz A, Justo P, Catalan MP, Sanz AB, et al. (2002). Apoptotic cell death in renal injury: the rationale for intervention. Curr. Drug Targets. Immune. Endocr. Metabol. Disord. 2: 181-192. 
Parra-Medina D, Messias DK, Fore E, Rachel M, et al. (2009). The partnership for cancer prevention: addressing access to cervical cancer screening among Latinas in South Carolina. J. S. C. Med. Assoc. 105: 297-305.

Semedo P, Wang PM, Andreucci TH, Cenedeze MA, et al. (2007). Mesenchymal stem cells ameliorate tissue damages triggered by renal ischemia and reperfusion injury. Transplant Proc. 39: 421-423.

Shahdadfar A, Fronsdal K, Haug T, Reinholt FP, et al. (2005). In vitro expansion of human mesenchymal stem cells: choice of serum is a determinant of cell proliferation, differentiation, gene expression, and transcriptome stability. Stem. Cells 23: 1357-1366.

Singaravelu K and Padanilam BJ (2009). In vitro differentiation of MSC into cells with a renal tubular epithelial-like phenotype. Ren. Fail. 31: 492-502.

Stolzing A and Scutt A (2006). Effect of reduced culture temperature on antioxidant defences of mesenchymal stem cells. Free Radic. Biol. Med. 41: 326-338.

Suga S, Kim YG, Joly A, Puchacz E, et al. (2001). Vascular endothelial growth factor (VEGF121) protects rats from renal infarction in thrombotic microangiopathy. Kidney Int. 60: 1297-1308.

Tang YL, Tang Y, Zhang YC, Qian K, et al. (2005). Improved graft mesenchymal stem cell survival in ischemic heart with a hypoxia-regulated heme oxygenase-1 vector. J. Am. Coll. Cardiol. 46: 1339-1350.

Zeisberg M and Kalluri R (2008). Reversal of experimental renal fibrosis by BMP7 provides insights into novel therapeutic strategies for chronic kidney disease. Pediatr. Nephrol. 23: 1395-1398.

Zhang YG, Yang Z, Zhang H, Wang C, et al. (2010). Effect of negative pressure on human bone marrow mesenchymal stem cells in vitro. Connect. Tissue Res. 51: 14-21.

Zwezdaryk KJ, Coffelt SB, Figueroa YG, Liu J, et al. (2007). Erythropoietin, a hypoxia-regulated factor, elicits a proangiogenic program in human mesenchymal stem cells. Exp. Hematol. 35: 640-652. 